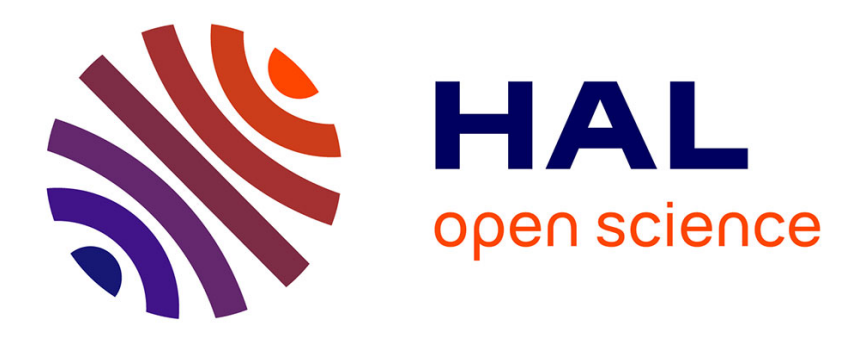

\title{
Modèles théoriques et définitions du nageur en France depuis 1960
}

Valérie Schwob, Hélène Joncheray

\section{To cite this version:}

Valérie Schwob, Hélène Joncheray. Modèles théoriques et définitions du nageur en France depuis 1960. STAPS : Revue internationale des sciences du sport et de l'éducation physique, 2013, 2 (100), pp.109-128. 10.3917/sta.100.0109 . hal-01563503

\section{HAL Id: hal-01563503 https://hal-insep.archives-ouvertes.fr/hal-01563503}

Submitted on 18 Jul 2017

HAL is a multi-disciplinary open access archive for the deposit and dissemination of scientific research documents, whether they are published or not. The documents may come from teaching and research institutions in France or abroad, or from public or private research centers.
L'archive ouverte pluridisciplinaire HAL, est destinée au dépôt et à la diffusion de documents scientifiques de niveau recherche, publiés ou non, émanant des établissements d'enseignement et de recherche français ou étrangers, des laboratoires publics ou privés. 


\title{
Modèles théoriques et définitions du nageur en France depuis 1960 Theoretical models and definitions of the swimmer in France since 1960
}

\author{
Valérie Schwob • Hélène Joncheray
}

Résumé : L'objet de cette étude est de mettre en évidence que l'évolution des définitions du nageur depuis 1960 varie en fonction des enjeux et des objets selon les époques et les institutions qui les génèrent. Les différentes institutions déclinent la définition du nageur en normes d'encadrement, ordonnant de ce fait les formes d'organisation de l'enseignement de la natation. À partir d'une analyse des textes officiels del'Éducation nationale, du ministère dela Jeunesse et des Sports et dela Fédération française de natation, nous serons conduits à associer ces définitions aux conceptions didactiques des institutions sous-jacentes. Les définitions du nageur varient et s'expriment par des tests. L'analyse des textes et tests nous montrera comment une convergence des objectifs des différentes instances s'est progressivement réalisée au cours de ces dernières décennies, s'exprimant partiellement à travers une définition similaire d'un savoir nager. Nous tenterons de démontrer qu'après une approche techniciste de la définition du nageur, les instances qualifient le nageur dans une conception globale s'inscrivant aussi bien dans le contexte culturel des pratiques que dans l'évolution pédagogique et didactique de l'activité.

motsclés: natation, éducation physique, didactique, savoirnager.

SummaRy: This article aims to formalizing the evolving of definitions of the swimmer since 1960. This paper develops the thought that this change depend from periods and institutions. The institution defines the scope of the swimmer through coaching and life guard staff. In fact the shaping of swimmer involves the swimming learning organization. Based on analyses of institutional instructions in physical education and Ministry of Sport we explain the link between swimmer definitions and didactic according with the institution underlying. Thus there are many definitions different tests and degrees. Recently they reflect the meeting between different swimming organizations. Thereby, the actually definition of the swimmer since similar. To sum up, after a technical method, the swimmer is considered in a global attitude who attests the practical cultural background. This change also stressed strongly the pedagogic and didactic evolutions.

KeywoRds: swimming, physical education, didactics, test.

\section{Zusammentassung: Theoretische Modelle und Definitionen des Schwimmers in Frankreich seit 1960}

Ziel dieser Untersuchung ist es aufzuzeigen, dass die verschiedenen Definitionen, von dem was ein Schwimmer ist, in ihrer Entwicklung seit 1960 je nach Epochen und Institutionen, die sie hervorbringen, und je nach Bedeutung und Gegenstand variieren. Die verschiedenen Institutionen nehmen die Schwimmer-Definitionen als Grundlage, um Normen für die Betreuung zu erstellen, und bestimmen damit die Organisationsformen des Schwimmunterrichts. Mittels einer Analyse der offiziellen Richtlinien des Bildungsministeriums, des Ministeriums für Jugend und Sport sowie des 
französischen Schwimmverbandes können wir diese Definitionen den didaktischen Konzeptionen der jeweiligen Institutionen zuordnen. Die Schwimmer-Definitionen variieren und werden durch Tests ausprobiert. Die Analyse der Texte und der Tests zeigt, dass sich in den letzten Jahrzehnten die Ziele der unterschiedlichen Instanzen allmählich angenähert haben, was sich teilweise in einer ähnlichen Definition des Schwimmen-Könnens zeigt. Wir werden versuchen aufzuzeigen, dass die Instanzen nach einem technischen Ansatz der Schwimmer-Definition nun den Schwimmer aus einer globalen Sicht betrachten. Diese Sichtweise ist sowohl im kulturellen Kontext der Praktiken als auch in der pädagogisch-didaktischen Evolution der Aktivität verankert.

SchlAgwöRteR: Schwimmen, Sportunterricht, Didaktik, Schwimmen-Können

\section{Riassunto: Modelli teorici e definizioni del nuotatore in Francia dopo il 1960}

L'oggetto di questo studio è di evidenziare che l'evoluzione delle definizioni del nuotatore dopo il 1960 varia in funzione delle poste in gioco e degli obiettivi secondo le epoche e le istituzioni che le generano. Le differenti istituzioni declinano la definizione del nuotatore in norme d'inquadramento, ordinando da questo fatto le forme d'organizzazione dell'insegnamento del nuoto. Partendo da un'analisi dei testiufficialidell'Educazione Nazionale, del Ministero della Gioventù e degli Sporte della Federazione Francese di Nuoto saremo portati ad associare queste definizioni alle concezioni didattiche delle istituzioni sottostanti. Le definizioni del nuotatore variano e si esprimono con dei test. L'analisi dei testi e dei test ci mostreranno come una convergenza degli obiettivi delle differenti istanze si è progressivamente realizzata nel corso di questi ultimi decenni, esprimendosi parzialmente attraverso una definizione similare della definizione del nuotatore. Tenteremo di dimostrare che secondo un approccio tecnicista della definizione del nuotatore, le istanze qualificano il nuotatore in una concezione globale iscrivendosi contemporaneamente sia nel contesto culturale delle pratiche sia nell'evoluzione pedagogica e didattica dell'attività.

PARole chiAve: didattica, educazione fisica, nuoto, saper nuotare.

\section{Resumen:Modelos teóricosy definiciones del nadador en Francia desde 1960}

Elobjetivo de este estudio es poner en evidenciala evolución delas definiciones del nadador desde 1960 varian en función de lo que está en juegoy de los objetos según las épocas ylas instituciones quelas generan. Las diferentes instituciones declinan la definición del nadador en normas de enmarcamiento, ordenando de esta manera las formas de organización de la enseñanza de la natación. A partir de un análisis de los textos oficiales de la Educación Nacional, del Ministerio de la Juventud ydelos Deportes y de la Federación Francesa de Natación, se conduciráala asociación de las definiciones hacia las concepciones didácticas sub yacentes de las instituciones. Las definiciones de los textos y de los test muestran como una convergencia de objetivos de las diferentes instancias se realizó progresivamente en el curso de los últimos decenios, exprimiéndose parcialmente una definición transversal similar al de un profesor de natación. Se busca demostrar que después de enfoque técnico de la definición de nadador, lasinstancias califican al nadador dentro de una concepción global inscribiéndose al mismo tiempo en el contexto cultural de las prácticas como también en la evolución pedagógica y didáctica de la actividad.

PalAbRAs claves : natación, educación física, didáctica, saber nadar. 


\section{1. intRoduction: sAvoiR nAgeR, une nécessité sociAle}

Nager est une des trois activités les plus pratiquées en France (Muller, 2005). Pratiquée à tout âge, cette activité est associée fréquemment à des faits tragiques liés aux risques inhérents à la pratique. Ainsi, l'Institut national de prévention et d'éducation pour la santé (INPES) et l'Institut national de veille sanitaire (INVS) fournissent des informations sur les noyades. Leur analyse montre un enjeu de l'apprentissage de la natation.

Tableau 1. Les noyades en France INPES,

relevés saisonniers du $1^{\mathrm{er}}$ juin au $1^{\mathrm{er}}$ octobre de 2003 à 2009

\begin{tabular}{|c|c|c|c|}
\hline & NOYADES & DONT DÉCÈS & $\begin{array}{c}\text { NOYADE CONCERNANT DES } \\
\text { ENFANTS DE MOINS DE 6 ANS }\end{array}$ \\
\hline 2003 & 1154 & 435 & $15,70 \%$ \\
\hline 2006 & 1207 & 401 & $15 \%$ \\
\hline 2009 & 1366 & 462 & $14 \%$ \\
\hline
\end{tabular}

Figure 1. Noyades accidentelles suivies ou non de décès selon l'âge des victimes, été 2003, France

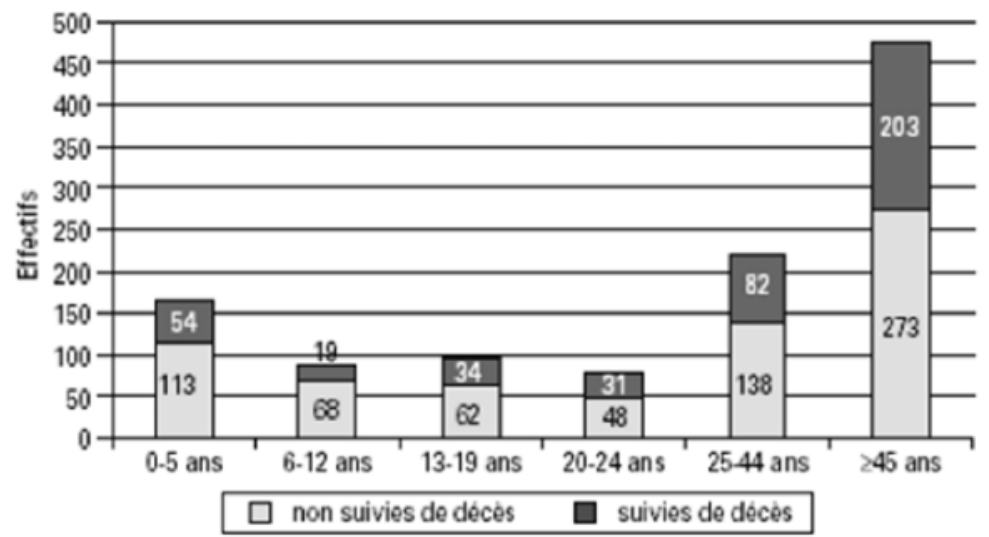

Source : enquête NOYADES 2003

Les causes de noyades accidentelles, propres à chaque âge, mettent en avant le fait que ne pas savoir nager est le premier facteur de risque de noyade chez les enfants de moins de 13 ans (43\%). Viennent ensuite le manque de surveillance (31\%) et les chutes (30\%) (De Maximy, 2004). En France, cet enseignement est organisé pour l'essentiel par le ministère de l'Éducation nationale etle ministère des Sports. Un des objectifs de l'Éducation nationale est de former des citoyens sachant nager, comme le précise le socle commun des compétences et connaissances (Loi 2005). Les programmes des collèges de 2008 définissent le premier degré du savoir nager (Arrêté du 8 juillet 2008, J.O. du 5 août 2008). Cependant, moins d'un quart des élèves scolarisés en France métropolitaine ne disposent pas d'un nombre de séances suffisant pour apprendre à nager, et $10 \%$ des élèves n'ont pas accès à cet enseignement pendant leur parcours scolaire (Freidland et al., 2009). Ainsi, le système fédéral, qui n'a pas pour projetdeformerl'ensemble dela population, complète toutefois l'offre de formation proposée par le ministère de l'Éducation nationale. Les différents dispositifs d'apprentissage agissent en complémentarité. Leurs limites respectives laissent toutefois une place à d'autres formes 
d'organisations, notamment privées. L'État est engagé à plusieurs niveaux dans cet enseignement. Des modifications récentes concernant les contenus des définitions du savoir nager, dans les deux ministères évoqués, montrent un intérêt institutionnel réel pour cette pratique. D'une part, la publication d'un texte officiel sur les normes d'encadrement et la sécurité de l'enseignement de la natation au sein de l'Éducation nationale (Circulaire n ${ }^{\circ} 2011$ 090 du 7 juillet 2011) rappelle les préoccupations sécuritaires liées à cette pratique au sein de l'école. C'est un élément du socle commun de compétence à acquérir lors de la scolarité. D'autre part, le ministère des Sports développe depuis 2006 une nouvelle définition harmonisée du savoir nager ainsi qu'une refonte de l'École de natation française. Au moyen de la création d'un Conseil interfédéral des activités aquatiques ${ }^{1}$, présidé par Francis Luyce administrateur du Comité national olympique et sportif français et président de la Fédération française de natation (FFN), l'objectif est d'établir une définition d'un " savoir nager " commune à seize fédérations sportives. Face à la multiplication des activités aquatiques (nage avec palmes, aquagym, nage en eaux vives, sauvetage, bébés-nageurs...) et la multiplication des fédérations sportives, ces enjeux du CIAA sont grands. Ce processus piloté par Patrick Gastou (DTNadjoint) etVincent Hamelin (FFN) doit permettre l'élaboration d'un test de référence du savoir nager en France. Il constitue la première étape d'un programme complet que ces fédérations devront progressivement mettre en place. Dans une même dynamique de lisibilité et afin de participer à la lutte contre les noyades mais également de favoriser l'accès aux différentes pratiques aquatiques, la Fédération française de natation est chargée, depuis 2008, de mettre en place une action massive d'apprentissage estival de la natation. L'enjeu de cette opération est d'offrir aux enfants le bagage essentiel qui leur permette d'assurer leur propre sécurité en milieu aquatique et de pratiquer les activités aquatiques en toute sécurité, identifié par le test du "Sauv'nage " de l'École de natation française. Il s'agit là d'éléments montrant la dynamique de l'enseignement de la natation française tant au point de vue du ministère des Sports que de l'Éducation nationale.

En France, savoir nager apparaît clairement comme un élément essentiel de la formation du citoyen. La définition du " savoir nager " permet de distinguer le nageur du non-nageur. Elle est une préoccupation récurrente des différents partenaires et atteste de la complexité et des enjeux qui s'y rattachent. En effet, définir le savoir-nager, c'est désigner un ensemble de spécifications décrivant une manière d'agir. Il en résulte parfois un standard sous forme de test servant de règle et de référence. Apparaissent dès lors certaines difficultés terminologiques, des divergences de points de vue et une pluralité des conceptualisations : le savoir nager sportif, le savoir nager sécuritaire, le savoir nager militaire... Les définitions expriment des approches culturaliste ou globale et reflètent des fondements théoriques, philosophiques et culturels différents. Au final, le caractère polythétique de cette notion se traduit par des constructions spécifiques de l'objet (Soulé \& Corneloup, 2007). Différentes définitions du savoir nager coexistent en France. L'Éducation nationale, la Fédération française de natation mais aussi d'autres fédérations à l'instar de la Fédération française de canoë-kayak, de voile, les scouts (Michel, 1942) ou encore l'armée ont établi une définition du savoir nager autour de tests spécifiques.

Considérant l'Éducation nationale et le ministère des Sports comme les principaux acteurs de l'enseignement de la natation, notre travail s'appuie sur une analyse historique des

1 CIAA:Conseil interfédéral des activités aquatiques crééen 2006, quicomprend 16 fédérations dont: la FFN, FNMNS, USEP, UNSS, UFOLEP, UGSEL, la fédération de triathlon, la fédération handisport, la fédération des clubs sportifs et artistiques de la défense, FFESSM, la fédération française de pentathlon moderne, la fédération française de sport adapté, la fédération française du sport d'entreprise, FFSU, FSCF, FSGT. Il comprend, en 2012, 4120000 licenciés. 
textes officiels de l'Éducation nationale et sur les tests proposés par la Fédération française de natation depuis 1960 .

Cetravaila pourobjectifde mettre en avant les différentes définitions du " savoir nager " afin d'interroger l'évolution des différents paramètres didactiques organisant l'enseignement de la natation en France.

Si aujourd'hui l'Éducation nationale et le ministère des Sports s'accordent en partie sur des critères d'une définition d'un savoir nager, nous montrerons que les positions et définitions prises par ces deux entités n'ont pas toujours reflété les mêmes objectifs. Ainsi, nous ferons l'hypothèse que la définition du savoir nager et des tests propres à cet apprentissage proviennent autant du traitement didactique de l'activité que des logiques institutionnelles et des enjeux culturels des époques concernées. Cette recherche s'appuie sur une revue de littérature portant sur les tests du savoir nager. Une comparaison entre les textes officiels de l'Éducation nationale sur le savoir nager et les définitions et tests produits par la Fédération française de natation sera envisagée en termes de distance à effectuer et d'acquisitions motrices aquatiques particulières.

Dans un premier temps, nous aborderons les pratiques fédérales au sortir des Jeux olympiques de Rome en 1960. Cette date constitue en effet un tournant dans la politique sportive (Arnaud, 1989, p. 260) s'illustrant par une nouvelle organisation de la vie sportive mais aussi par la mise en place des équipements sportifs. Nous verrons comment l'élaboration de la définition du savoir nager a évolué à partir de cette époque au gré des transformations de la société. Dans un deuxième temps, nous nous attarderons sur les définitions données dans le cadre del'Éducation physique et sportive (EPS). Tentant de saisir les évolutions didactiques depuis les années 1960, nous évoquerons particulièrement 1965, date d'une circulaire sur la natation qui fera référence pendant presque 40 ans. La natation est une activité de l'éducation physique depuis la fin du XIX $^{\mathrm{e}}$ siècle (Pelayo \& Terret, 1994). Le dernier demi-siècle (1960-2011) s'illustre par des changements conséquents d'appartenance ministérielle et de reconnaissance disciplinaire. Les débats entre la sportivisation de la discipline et la formation de base de l'individu (During, 1981) occasionnent un questionnement sur les pratiques de terrain liées à l'enseignement de la natation en EPS (Auvray, 2010). Enfin, nous serons amenées à discuter des influences réciproques de ces différentes instances dans un contexte d'évolution des pratiques sportives et deloisirs.

\section{SAvoiR nAgeR}

Nager et apprendre à nager se définissent culturellement et historiquement. Dès 1934, Marcel Mauss l'exprimait lors d'une communication présentée à la Société de psychologie: "Je savais bien quela marche, la nage, par exemple, toutes sortes de choses de ce type sont spécifiques à des sociétés déterminées ; que les Polynésiens ne nagent pas comme nous, que ma génération n'a pas nagé comme la génération actuelle nage " (Mauss, 1950, p. 366).

Définir la notion du savoir nager fait apparaitre les enjeux qui contribuent à son élaboration. La problématique d'une définition réside dans les choix qu'elle implique. Elle établit ainsi une structure ordonnée, une arborescence par niveau des éléments à acquérir, elle oriente didactiquement les acquisitions attendues. La définition du savoir nager n'est jamais absolue mais relative culturellement, socialement et historiquement (Catteau, 1999). Cette étape est souvent associée à la définition d'une tâche finale illustrant la compétence à démontrer. Nous tenterons de l'appréhender à travers les tests qui balisent les étapes d'apprentissage et qui en filigrane laissent apparaître des conceptions variées de la natation. Ainsi, la natation, abordée initialement dans un cadre hygiéniste et sécuritaire (Terret, 2002), se voit 
progressivement considérée comme une activité physique et sportive au même titre que les autres dans le second degré de l'enseignement. Intégrée depuis 1877 dans les programmes de l'éducation physique (Pelayo \& Terret, 1994), l'apprentissage initial de la natation relève plus spécifiquement du premier degré del'enseignement, des structures associatives ou de structures privées. Nous aborderons dans un premier temps les définitions fédérales et leurs évolutions puis nous envisagerons les définitions scolaires.

\subsection{Savoir nager dans le monde fédéral}

Certaines fédérations sportives (les fédérations françaises de ski, de judo, de natation par exemple) organisent leurs pratiques au travers de brevets et de tests qui attestent de la progression sportive et motivent le pratiquant. Ceux-ci permettent également une plus grande lisibilité des contenus et la création d'un patrimoine culturel commun. En natation, un brevet élémentaire scolaire de natation existe dès les années 1920 puisqu'en 1921 la FFNS a délivré 1911 brevets (Terret, 1998). Il consiste en un départ plongé suivi d'un parcours de 25 mètres en nage libre. Il sera repris en 1938 lors de la mise en place du Brevet sportif populaire créé en 1937 (Andrieu, 1990, p. 64). Il s'agit alors de mesurer une performance. L'objectif de ce brevet est de remettre le citadin au contact de la nature, de favoriser l'émancipation, la démocratisation de la pratique physique, la santé et l'éducation. L'épreuve de natation est facultative en raison du faible nombre d'infrastructures. Le test de natation du Brevet sportif populaire fait office dès lors d'attestation du savoir nager. Après une description des tests, nous évoquerons les définitions successives du savoir nager données depuis 1960 par la Fédération française de natation.

Dans les années 1960, trois conceptions de la natation coexistent. La natation éducative de l'École normale supérieure de l'Éducation physique (ENSEP) a pour objectif d'écarter le risque de la noyade chez l'enfant. Au moyen d'une gymnastique rythmée et rationnelle, elle permet de développer sa morphologie, ses grandes fonctions, son caractère et son sens social (Schoebel, Raude, \& Bécart, 1952). Si l'enseignement s'organise autour de l'apprentissage de la brasse à travers la maitrise de la respiration, de la propulsion et de l'équilibration (Terret, 2006), savoir nager reste défini par une distance à franchir en brasse traditionnelle, tête hors de l'eau. La natation sportive de la Fédération française de natation s'envisage essentiellement autour des techniques de nage sportive. Savoir nager consiste en un parcours dans une nage. Dans une dynamique sportive, Raymond Catteau, professeur d'EPS et conseiller technique régional de natation propose une troisième conception de la natation. Ce pédagogue tente de dégager un dénominateur commun en termes de principes invariants d'équilibre, de respiration et de propulsion (Erard \& Catteau, 2008). Savoir nager, pour ce théoricien, s'exprime dans un idéal inaccessible qu'il formulera plus tard par la résolution de la triple problématique d'un meilleur équilibre de la meilleure propulsion et de la meilleure respiration dans l'élément liquide (Catteau \& Garroff, 1968, p. 71).

Ces conceptions expriment des usages différents du savoir nager. Face à cet éclectisme de conception (sécuritaire, sportive, globale), savoir nager s'atteste au travers de la capacitéà franchir une distance (25 mètres) sans prendre appui dans une forme de nage codifiée. La brasse est la technique utilisée, elle est héritée de la méthode française de 1920 (Terret, 1998). Chez Émile Schoebel, la natation est développée sous une forme globale après une longue étape de familiarisation (Terret, 2006). La FFN développe des tests des niveaux distincts caractérisés par des distances différentes ( $25 \mathrm{~m}, 50 \mathrm{~m}, 400 \mathrm{~m})$. Ces tests sont utilisés pour construire des étapes d'apprentissage à visée sportive et utilitaire. Des brevets, décernés par les maitres-nageurs officialisent une compétence à savoir nager, reconnue nationalement. 


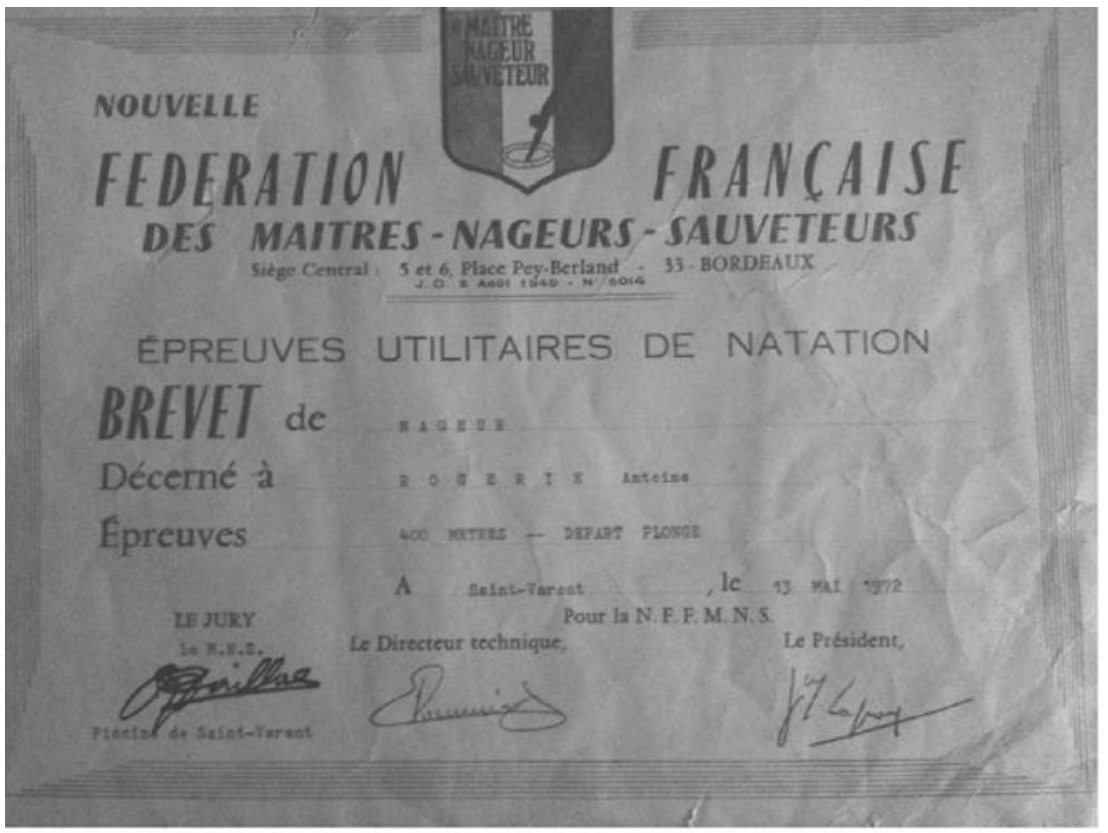

La construction de ces étapes aquatiques se situe dans une période de changement structurel de la population et de la société. Dans les années 1960 se profile une modification du rapport au corps, aux loisirs et aux sports. La population active est plus importante, la place des femmes dans la société est en pleine mutation, d'autre part la place du travail et du temps libre dans la société s'organise (Clément, 1994, p. 147). Le nombre de licenciés (pratiques sportives) de la Fédération française de natation passe de 34307 licenciés en 1960 pour 688 clubs à 68406 en 1970 pour 989 clubs (source département statistiques FFN). La massification des pratiques sportives se réalise dans un temps de confrontation non armée. La guerre froide s'opérationnalise à travers les rencontres sportives. L'internationalisation du sport est l'occasion de prouver l'efficacité des systèmes en compétition. Dans ce cadre, les brevets et tests participent à l'organisation de structures cohérentes, à la diffusion et au rayonnement des pratiques sportives. Au point de vue structurel, le IV Plan (1961-1965) développe les équipements sportifs avec la première loi-programme (Mille piscines, milles stades et terrains de jeux). En 1961, elle permet la construction d'environ 1000 gymnases, 250 piscines, 2200 stades et terrains de sport (Callède, 2000). Lors du Ve Plan (1966-1970), réalisé par Jean-Baptiste Grosborne, nageur du Club des nageurs de Paris, une seconde loi-programme suit avec cette fois-ci une grille d'implantation de ces équipements, traduisant un réel effort de normalisation. Des modèles de piscines " tournesol ", " caneton " sont inventés, associant des espaces ludiques aux bassins sportifs et rectangulaires traditionnels. Le sport est à l'époque associé à la production banale de l'urbain, surtout pour les petites et moyennes villes dans un esprit valorisant le sport de masse àl'échelon national(Merle, 2008).

\subsection{Les tests du monde fédéral}

Dans ce contexte de développement des pratiques sportives (Artiaga, 2007, p. 109), le secrétariat d'État auprès du Premier ministre, chargé de la Jeunesse, des Sports et des Loisirs met 
en place en 1971 une échelle de tests (ENSEPS, 1973). Ces épreuves constituent une progression et sanctionnent des degrés de qualification à la fois sur le plan de la natation et sur celui du sauvetage. De fait, ces tests sont inspirés des capacités à savoir nager, à savoir se prémunir contre les risques de noyade, et à pouvoir nager. Le "canard "etle "têtard" sont ajoutés etdes insignes et cartes sont délivrés (Comiti, circulaire 1971). L'ensemble de ces tests (canard, têtard, dauphins et tritons ${ }^{2}$ ) et de leurs différents niveaux (par exemple : dauphin d'or ${ }^{3}$, dauphin d'argent, $1^{\mathrm{er}}, 2^{\mathrm{e}}, 3^{\mathrm{e}}$ tritons) " constituent " alors l'École de natation française. Ces tests de la FFN évaluent un savoir-faire sportif puisqu'il s'agit de franchir une distance dans une technique de nage, mais également des dimensions sécuritaires (remorquage). Ils illustrent la progression pédagogique de l'École de natation française. Les tests des " tritons " sont passés dans les établissements scolaires sous l'autorité du chef d'établissement et assistés d'au moins un professeur d'EPS. Dans le cadre scolaire, des sessions sont également organisées sous la responsabilité d'un jury présidé par le président du club assisté d'au moins un maitre-nageur sauveteur. Le ministère de l'Éducation nationale et le ministère de la Jeunesse et des Sports coopèrent donc pour enseigner et développer la pratique dans une même acception. Dans les années 1990, alors que l'EPS confirme une orientation plus éducative que sportive, une refonte des tests ENF aboutit à la création du passeport de natation. Celui-ci s'organise autour de tests appelés les " dauphins " de différentes couleurs selon le niveau (arc-en-ciel $\left.{ }^{4}\right)$. Les évaluations des tests ont lieu dans les clubs de natation affiliés à la Fédération française de natation, ils évaluent une compétence sécuritaire et utilitaire reflétant l'évolution des pratiques vers un sport pour tous (Artigia, 2007, p. 113). En 2008, ce sont les " pass'ports de l'eau " et " pass'compétition " qui prennent la suite des "dauphins ", créant une certaine rupture conceptuelle. Ils consistent en différents tests issus de la nage avec palmes, du water-polo, du plongeon, de la natation synchronisée ainsi que dela natation de course. Ces tests concernent les enfants inscrits dans des clubs affiliés à la Fédération française de natation, ils se déroulent dans des sessions organisées localement par le comité régional ou départemental de la Fédération française de natation. Ils ont pour vocation de développer l'image des autres disciplines de la FFN, de diversifier les pratiques de jeunes apprentis vers d'autres compétences aquatiques que la nage sportive. Le conseil interfédéral des activités aquatiques au travers de l'école de natation française propose une formation du nageur en trois étapes. Les deux premières étant constituées par le sauv'nage etle pass'sports de l'eau. Ces tests s'attachentà développer les pratiques fédérales et à s'adapter aux évolutions des pratiques culturelles. De fait, la formation du nageur devient plus riche et plus variée. Les tests de l'école de natation ont évolué d'une vocation sécuritaire et sportive dans les années 1970, et se déclinent actuellement autour d'une motrice aquatique variée et d'une ouverture culturelle sur les activités aquatiques. L'aspect sécuritaire reste néanmoins un pré-requis à la pratique d'activité aquatique.

Si l'ENF a évolué progressivement durant ce demi-siècle, il n'en est pas de même pour le savoir nager. En 1960, savoir nager est validé par le Brevet de Natation. Ce diplôme officiel est délivré par un maître-nageur et/ou un entraîneur. Il atteste qu'un individu est capable de nager une certaine distance $(25 \mathrm{~m}, 50 \mathrm{~m}$, 100 m, 200 m, 400 m). Ce diplôme permet

\footnotetext{
$23^{e}$ triton: parcourir $200 \mathrm{~m} 4$ nages départ plongé. Départau borddu bassin, rechercherun figuratifimmergéà $2 \mathrm{~m}$, leremonterà la surface et le transporter sur $25 \mathrm{~m}$.

3 Dauphin d'or: réaliserun $400 \mathrm{~m} 4$ nages départplongé en 8 minutes pour les garçons et 8'40 pour les filles. Parcourir 25 mètres, aller chercher un objet à 2 mètres, le remonterà la surface et le remorquer sur 25 mètres en moins de 1'30 (garçons) et 1'40 (filles). 4 Dauphin blanc : saut du bord de la piscine, maintien libre sur place 5 secondes avec une planche, parcourir 10 mètres. Dauphin vert : avec un tee-shirt, réaliser $21 \mathrm{~m}$, passer sous un obstacle, faire demi-tour, enlever le tee-shirt et finir le $50 \mathrm{~m}$.
} 
entre autres d'attester d'une compétence en vue de la pratique d'activités nautiques et de plein air. Si la formation du nageur a subi des modifications de contenu, le test du " savoir nager " n'a pas ou peu évolué depuis 1960. En 2006, le Conseil interfédéral des activités aquatiques, conçu pour promouvoir et développer les activités aquatiques, a mis en place une réflexion pour définir un savoir nager commun aux différentes fédérations s'occupant d'activités aquatiques. Un nouveau qualificatif a été trouvé, il s'agit du test du " sauv'nage " ${ }^{5}$. Ce test est défini autour de huit tâches à enchaîner. L'objectif est d'harmoniser les définitions du savoir nager entre les différentes fédérations, d'établir une cohérence nationale, ainsi qu'une plus grande lisibilité. Ce test constitue à lui seul un changement considérable, les modèles culturels des différents acteurs sont alors effacés au profit d'une définition globale par les différentes fédérations. Symbolisant une définition unitaire, le Sauv'nage occasionne une modification didactique de l'objet. Ce test délaisse les techniques de nages sportives au profit d'une adaptation au milieu. L'apprentissage de la natation prend en compte les nouvelles pratiques aquatiques (eaux vives, activités nautiques, ludiques...), et intègre dans son évaluation des compétences globales. Le sauv'nage est adapté à tout public. Il agrège l'évolution des conceptions des activités aquatiques (d'éveil, de loisir, d'entretien) témoignant d'une nouvelle " philosophie de l'eau " associée à une nouvelle représentation de l'enfant amorcée depuis 1968 (Garnier, 1995, p. 211).

Parallèlement au test du sauv'nage illustrant l'harmonisation des conceptions et le renouveau de l'École de natation française, une action de grande envergure est mise en place par le ministère des Sports en 2008 : l'opération
" savoir-nager " pilotée par V. Hamelin de la Fédération française de natation. Au-delà de la prévention active des noyades, le "SavoirNager " est considéré par le ministère des Sports comme un véritable enjeu de société. Il s'agit de transmettre le bagage essentiel permettant de pratiquer des activités aquatiques en toute sécurité, identifié par le test du Sauv'nage de l'École de natation française. Les compétences visées participent à une nouvelle définition de la natation orientée vers une réelle adaptation aquatique. Cette opération exprime à la fois une dimension humaniste à travers l'accès à l'apprentissage et une stratégie politique de développement des pratiques fédérales. Savoir nager induit un accès à la culture de l'eau et à toutes les activités aquatiques existantes disponibles pour les enfants, la natation, mais aussi les activités nautiques (voile, plongée, canoë, jeux d'eau, etc.). En 2010, l'opération " savoir nager " du ministère des Sports s'est déroulée dans 105 communes pour 3113 participants (tout public) alors qu'en 2008 seulement 1541 participants avaient pu bénéficier de cet enseignement. En moyenne, en 2010, 65 \% des participants réussissent le test du sauv'nage. Cette opération est le résultat d'une organisation considérable. Ainsi, les associations ou les collectivités participantes doivent présenter des pré-requis : elles doivent être en capacité de proposer un programme d'apprentissage de la natation pendant les vacances d'été sous forme de stage d'apprentissage de quinze séances d'une heure à raison d'une séance par jour cinq jours par semaine. Cette forme d'organisation en séances rapprochées rejoint les propositions faites par l'Éducation nationale dans sa circulaire de 2011 (Circulaire ${ }^{\circ} 2011-090$ du 7 juillet 2011) et illustre une forme classique de l'enseignement de la natation sous forme de

\footnotetext{
5 Testdu sauv'nage: sauter du bord, se laisser remonter passivement / réaliser unéquilibre en position ventrale / nager jusqu'au cerceau ety entrer / rester en position verticale tête hors del'eau durant 5 secondes / ressortir du cerceau, nager (entre 15 et $20 \mathrm{~m}$ ) / passer sous chaque obstacle (exemple: lignes d'eau) 3 ou 4 espacés d'au moins 1,50 m sans reprendre d'appuiau mur ou sur laligne d'eau / réaliser un équilibre en position dorsale / nager sur le dos (entre $15 \mathrm{~m}$ et $20 \mathrm{~m}$ ) / à la fin du parcours, aller chercher un objet au fond du bassin / lemontrer au-dessus del'eau / lelâcher, terminer le parcours en position dorsale. Le sauv'nage aété élaboréavec la Fédération handisport, la Fédération d'éveil... et de cefaita été validé pour différents publics (personnes âgées, personnes à mobilitéréduite...).
} 
stage de dix ou quinze leçons, forme fréquente de l'apprentissage estival de la natation en piscines municipales ou privées ou dans les clubs de plage (Gresser, 2005).

\subsection{Savoir nager à l'école}

En $1959^{6}$, la natation scolaire passe d'un enjeu de formation minimale disciplinaire à une conception plus utilitaire et fondamentale. Les instructions officielles de 1959 intègrent la natation au sein des séances de plein air, c'està-dire en dehors de la leçon proprement dite, sans doute en raison du manque d'infrastructures et des influences de la méthode naturelle de Georges Hébert à l'égard des bienfaits de l'air et du soleil (Terret, 1996).

Dans des années 1960, la sportivisation globale des enseignements oriente l'enseignement de la natation dans une dimension sportive au niveau du curriculum formel (Auvray, 2010). En raison des enjeux sécuritaires, l'enseignement de la natation s'effectue avec des effectifs réduits. En 1965, la parution d'un texte officiel concernant l'encadrement de la natation (B.O. $\mathrm{n}^{\circ} 6$ ) fait référence pendant près de quarante ans bien que des aménagements apparaissent progressivement. Dans ce texte, savoir nager se définit comme la capacité à franchir une distance de cinquante mètres sans reprise d'appui. Les dimensions sécuritaires définissentl'organisation de l'enseignement de la natation. Ainsi, le texte précise que l'enseignement doit s'effectuer avec des effectifs réduits : "Le nombre d'élèves apprenant à nager doit être limité à 16 au maximum quand il s'agit de débutants ou d'élèves susceptibles de ne parcourir que moins de 50 mètres en eau profonde et de 20 à 24 élèves pour les nageurs nageant aisément au moins 50 mètres [...]. Cet enseignement exige un minimum de deux enseignants par classe lorsque l'effectif des élèves (dispensés exclus) dépasse 16... " L'usage de la brasse répond à un enjeu sécuritaire, celui de pouvoir rejoindre le bord, et aux pratiques culturelles liées au développement des pratiques balnéaires (Artiaga, 2007, p. 109). La programmation des activités physiques et sportives dans les établissements du second degré, publiée conjointement parle ministère de l'Éducation nationale et le ministère de la Jeunesse et des Sports en 1967, s'appuie sur les travaux de Raymond Catteau. Le passage de tests ou de brevets décrits dans la programmation de 1967 sanctionne les niveaux en natation utilitaire, natation sportive et sauvetage. L'apprentissage de la natation est essentiellement une adaptation progressive au milieu aquatique (indissociable d'une maitrise de la respiration), obtenue par un travail principalement global, dynamique et foncier et finalisé par l'apprentissage des nages sportives et de l'entraînement.

Dans les années 1970, dans un contexte de développement des loisirs (Sobry, 2004), la mise en place des classes de mer et de séjour en plein air dans les établissements du premier degré (Garnier, 2002) invite à développer la pratique de la natation dans le cadre scolaire. La circulaire du 23 décembre 1971 témoigne de l'essor de l'activité et précise que "la meilleure organisation pédagogique à l'école élémentaire est réalisée lorsque s'ajoutent aux instituteurs des classes concernées d'autres éducateurs : maitres-nageurs sauveteurs (MNS), moniteurs municipaux qualifiés, éducateurs sportifs, etc., de façon à tendre vers la proportion de deux enseignants par unité de 30 enfants environ ". Une augmentation marquée des effectifs, passant en 1958 de 1174400 élèves à 3165600 en 1975 (Viger, 2007) aboutit en 1975 à la Loi Haby créant le collège unique. Sous le poids de ces effectifs, les méthodes vont progressivement se modifier. Dans un contexte de massification de l'enseignement (Merle, 2002), la natation s'oriente effectivement vers l'acquisition d'une aisance aquatique et l'acceptation du milieu dépassant ou délaissant progressivement

6 Pour G. Hébert, en 1959, savoir-nager consiste à " flotter sans mouvements ou avec mouvements réduits, pouvoir franchir une distance, évoluer de diverses façons en surface et en plongée, sauter par la tête ou les pieds, assurer sa propre sécurité, porter secours, exécuter ce quiprécèdeen étant habillé, faire face auxincidents et dangers des eauxen pleine nature »(Terret, 2006, p. 30). 
le cadre purement technique d'une natation sportive. L'approche se veut globale, associant les grands principes de respiration d'équilibre et de propulsion, mais l'enseignement de la natation reste finalisé par l'acquisition des quatre nages et de l'entraînement.

Cette approche témoigne de l'influence d'enseignants maitres-nageurs tels Raymond Catteau, notamment lors des stages Maurice Baquet entre 1965 et 1975 (Goirand, Journet, Marsenach et al., 2004, p. 221).

Une centration sur l'enfant (Piaget, 1972), exprimée dans des expériences aquatiques des bébés nageurs (Vadepied, 1976), contribue également à la modification de l'approche de l'activité. Elle se fait plus précocementets'éloigne du concept d'efficacité sportive au profit d'un éveil sensori-moteur.

Durant les années 1980, les lois de décentralisation occasionnent une territorialisation de l'action éducative (Garnier, 2002). Une critique techniciste des praticiens participe alors à la dénonciation des intervenants sportifs extérieurs. Dans ce contexte conflictuel, l'institution scolaire affiche clairement, au travers de l'arrêté 1985, une politique " volontariste " d'ouverture des seconds cycles généraux, technologiques et professionnels. L'objectif du ministre Jean-Pierre Chevènement, selon lequel " $80 \%$ des jeunes d'une classe d'âge doivent accéder au baccalauréat " pour l'an 2000, est affiché (Loi nº 89-486 du 10 juillet 1989). L'enseignement " de masse " va donc devoir tenir compte de la forte hétérogénéité des élèves. Dans ce contexte, les programmes et instructions du collège (Arrêté du 14 novembre 1985, paru au B.O. du 12 décembre 1985) précisent que l'apprentissage de la natation en classe de sixième consiste en " une adaptation progressive au milieu aquatique permettant un rapide accès aux nages de compétition. La mâ̂trise de cette discipline et du milieu dans lequel elle s'exerce est en relation directe avec la maîtrise de la respiration. "Aucune définition du nageur n'est développée et une approche globale de la natation est sous-tendue, identique à celle de 1967. Nous constatons dans les textes un abandon de la référence à l'apprentissage du geste au profit d'une maitrise du milieu aquatique ("maitrise du comportementet des déplacements dans l'eau, maitrise d'évolutions diverses sur l'eau et sous l'eau, plongeons élémentaires "), d'une véritable " possession du monde ", élément constant depuis les années 1945 mais décliné plus spécifiquement (Pelayo \& Terret, 1994). L'enseignement semble alors libéré du carcan des conceptions et du conditionnement mécanistes de l'apprentissage des techniques de nage sportive, bien que l'objectif d'une "initiation aux quatre nages sportives " demeure in fine.

La Loi d'orientation de 1989 place l'élève au centre des apprentissages. Cette centration sur l'élève et ses besoins s'appuie sur le traitement didactique de la natation tel qu'il est envisagé dès 1986, c'est-à-dire dans sa forme globale. L'apprentissage initial est davantage axé sur une adaptation au milieu aquatique que sur l'acquisition rigoureuse des techniques de nage.

La réforme des horaires des collèges annoncée par le nouveau contrat pour l'école (Loi $n^{\circ}$ 95-836 du 13 juillet 1995) amène une redéfinition du volume d'heures d'éducation physique et sportive. Une quatrième heure est attribuée aux classes de sixième leur permettant d'accéder notamment à un apprentissage de la natation. Mais il faudra attendre 2004 pour qu'une nouvelle circulaire natation apparaisse etaccordeles moyens des " priorités " affichées avec la quatrième heure d'EPS.

En 1996, les textes officiels institutionnalisent une nouvelle définition du savoir-nager avec la mise en place des nouveaux programmes pour les sixièmes. Celle-ci officialise une conception didactique de la natation et des pratiques pédagogiques dominantes des enseignants d'EPS. Cette définition présente un premier tournant conceptuel. Un élève sachant nager est alors capable, en eau profonde, 
de sauter ou plonger pour réaliser sans interruption une distance donnée, un maintien sur place et la recherche d'un objet immergé (B.O. $n^{\circ} 29$ du 18 juillet 1996).

L'évolution de cette définition confirme une conception globale du nageur et de l'enfant dans une démarche qui demeure toutefois sécuritaire. Il s'agit alors de former un citoyen adaptable et non plus un technicien spécialisé. Durant cette décennie, des propositions similaires de tests définissant le savoir nager foisonnent (Mestejanot, 1992 ; GalPetitfaux, 1993 ; Gourson-Verger \& Verger, 1994 ; Maillard \& Pelayo, 1994). La définition du savoir nager scolaire illustre alors l'évolution des conceptions pédagogiques et didactiques mais évoque aussi la stratégie identitaire de l'EPS. Une transposition didactique des savoirs savants illustrés par les activités sportives fédérales en savoirs scolaires, disciplinaires organisent l'enseignement de l'EPS. L'activité aquatique est prise dans son ensemble, il faut être adaptéàl'eau pour pouvoir s'y mouvoir librement. La finalité devientune autonomie de déplacement et d'évolution aquatique et non plus une technique codifiée illustrant une autonomie. Ces objectifs sont sous-tendus par des conceptions de l'enfant qui le placent en tant qu'acteur de ses apprentissages.

En 2000, un test est rendu obligatoire à l'écoleélémentaire pour la pratique des sports nautiques (Circulaire n ${ }^{\circ}$ 2000-075 du 31 mai 2000). Il s'agit d'un test " anti-panique ". Il consiste à l'enchaînement de trois tâches (entrée dans l'eau, déplacement, passage sous une ligne) sur une distance de 20 mètres minimum réalisé habillé. L'arrivée de ce test, à toute fin juridique, illustre également une conception globale de l'apprentissage orienté dans une direction sécuritaire. Il nous permet de confirmer que les préoccupations éducatives concernent alors les activités de plein air, désormais parties intégrantes des activités scolaires et de ce fait finalisant l'apprentissage d'un savoir nager.

En 2004, une modification importante des conditions d'enseignement de la natation est définie (Circulaire n² 2004-13 du 13 juillet 2004). Cette circulaire fixe des normes d'encadrement et des effectifs contraignants. Ces données auront pour conséquence de réduire les effectifs des groupes de non-nageurs et de renforcer l'encadrement de l'activité scolaire. Le niveau d'autonomie en fin d'école élémentaire est évalué par un parcours d'environ 15 mètres en eau profonde, sans brassière et sans appui. L'objectif à l'école primaire n'est pas d'apprendre à nager, mais de développer des compétences qui permettent une autonomie motrice aquatique.

La circulaire de 2004 définit également différentes étapes d'apprentissage en termes d'autonomie de déplacement : quinze mètres pour l'enseignement élémentaire, et pour le secondaire cinquante mètres ou encore un test inspiré du test " pechomaro " (Maillard \& Pelayo, 1994) qui consiste en un enchaînement de sept tâches sur un parcours de 50 mètres en eau profonde. Cette circulaire commune pour le primaire et le secondaire (Touchard, 2004) remplace celle de 1965 et transforme l'organisation de la natation scolaire du secondaire, permettant la mise en place de très petits groupes de non-nageurs (12 élèves et moins).

Ce texte décline différents niveaux du savoir nager (moins de 15 mètres, entre 15 et 50 mètres, et au-delà de 50 mètres). Si ce texte occasionne des demandes de moyens horaires supplémentaires souvent attribuées par les chefs d'établissement, il illustre aussi le nombre résiduel de non-nageurs et la complexité de cet enseignement. Le fait qu'un nombre conséquent d'élèves entre au collège sans avoir atteint les compétences attendues en fin de CM2, éclaire l'attention portée. La coïncidence réelle ou fortuite de cette nouvelle circulaire au moment d'un rayonnement international de la natation française aux Jeux olympiques d'Athènes, réorganise et renforce l'enseignement de la natation. Cette dynamique sera confirmée par la loi du 23 avril 2005 d'orientation et de programme 
pour l'avenir de l'École. Définissant le socle commun de connaissances et de compétences, elle place la capacité à savoir nager dans la compétence autonomie et initiative du socle (Décretdu 11 juillet2006, p. 23).

En 2011, la dernière publication de l'Éducation nationale concernant la natation rappelle la répartition de cet apprentissage sur les trois paliers du socle commun (B.0. 28 du 14 juillet
2011). Le savoir nager visé au dernier palier du socle commun est défini dans les programmes du collège par le " premier degré du savoir nager ". Il correspond à une maîtrise du milieu aquatique permettant de nager en sécurité dans un établissement de bains ou un espace surveillé. La circulaire rappelle la construction du savoir nager durant la scolarité. Elle s’inscrit autour de paliers distincts.

\section{Tableau 2. Définition des paliers successifs du savoir nager dans l'enseignement scolaire français}

\begin{tabular}{|c|c|c|}
\hline Testanti-panique & $\begin{array}{l}\text { Test à réaliser } \\
\text { en cas d'activité } \\
\text { nautiqueàl'école } \\
\text { élémentaire. }\end{array}$ & $\begin{array}{l}\text { Départ par une chute arrière volontaire à partir d'un } \\
\text { tapis disposé dans l'eau puis déplacement dans l'eau sans } \\
\text { présenter de signe de panique surun parcours de } 20 \mathrm{~m} \text {, } \\
\text { avec passage sous uneligne d'eau, posée et non tendue } \\
\text { dans la partie d'un bassin d'une profondeur au moins égale } \\
\text { à } 1 \mathrm{~m} 80 \text {. }\end{array}$ \\
\hline Le premier palier & $\begin{array}{l}\text { À atteindre } \\
\text { en fin de cycle } 2 \\
\text { (CE1; } 7 / 8 \text { ans) }\end{array}$ & $\begin{array}{l}\text { Undéplacement surunequinzaine demètres, une } \\
\text { immersion et un déplacement sous l'eau, ainsi qu'une } \\
\text { flottaison. }{ }^{\text {i }}\end{array}$ \\
\hline Le deuxième palier & $\begin{array}{l}\text { En fin de cycle } 3 \\
\text { (CM2 10/11 ans) } \\
\end{array}$ & $\begin{array}{l}\text { Un déplacement surune trentaine de mètres, un plongeon, } \\
\text { uneimmersion et un déplacement } \mathrm{t}^{\mathrm{ii}} \text {. }\end{array}$ \\
\hline Le troisième palier & $\begin{array}{l}\text { Classe de sixième } \\
(11 / 12 \text { ans }) \text { ou } \\
\text { fin de troisième } \\
(15 \text { ans })\end{array}$ & $\begin{array}{l}\text { "Premier degré du savoir nager" } \\
\text { Sauter en grande profondeur, reveniràla surface et } \\
\text { s'immerger pour passer sous un obstacle flottant, nager } \\
20 \text { mètres : } 10 \text { mètres surleventre et } 10 \text { mètres surledos, } \\
\text { réaliser un sur-place de } 10 \text { secondes, s'immerger à nouveau } \\
\text { pour passer sous un obstacle flottant. Ainsi que des } \\
\text { connaissances et attitudes essentielles à vérifier: connaître } \\
\text { les règles d'hygiène corporelle, connaîtreles contre- } \\
\text { indications, prendre connaissance du règlement intérieur } \\
\text { del'installation nautique, connaître etrespecter le rôledes } \\
\text { adultes encadrants. } \\
\text { Le premier degré du savoir nager constitue une } \\
\text { compétence à nager en sécurité, dans un établissement de } \\
\text { bains ou un espace surveillé (piscine, parc aquatique, plan } \\
\text { d'eau calme à pente douce). }\end{array}$ \\
\hline Lequatrième palier & Fin de troisième & $\begin{array}{l}\text { Le savoir nager deuxième degré est un enchaînement de } \\
\text { six tâches dont un déplacement de } 10 \text { minutes sans appuiii. } \\
\text { "Deuxième degré du savoir nager " }\end{array}$ \\
\hline
\end{tabular}

iPalier 1: Se déplacersurunequinzaine de mètres sans aideàlaflottaisonet sans reprised'appuis. Et effectuerun enchaînementde 3 actions sans reprise d'appuis, en moyenne profondeur, amenant à s'immerger en sautant dans l'eau, à se déplacer brièvement sous l'eau (par exemple pour passer sous un obstacle flottant) puis à se laisser flotter un instant avant de regagner le bord.

ii Palier 2 : Se déplacer sur une trentaine de mètres sans aide à la flottaison et sans reprise d'appuis. Par exemple, se déplacer sur 25 mètres, effectuer un virage, une coulée et une reprise de nage pour gagner le bord; enchaîner, sans reprise d'appuis, un saut ou un plongeon en grande profondeur, un déplacement orienté en immersion (par exemple pour passer dans un cerceauimmergé) et un sur-place de 5 à 10 secondesavant de regagner le bord.

iii Palier 4 : 1) plongeon, 2) nage 10 m en immersion, 3) nager 10 minutes sans prendre appui, 4) maintien sur place 1 minute, 5) effectuerun plongeon canard pourrechercherun objetet6)remorquercet objet sur 20 m surledosles bras croisés surla poitrine.

Dans un contexte scolaire d'éducation à la citoyenneté et la santé (Circulaire n 2006197 du 30 novembre 2006), des connaissances et attitudes relatives aux règles d'hygiène et de sécurité propres aux établissements de bains et aux activités aquatiques s'ajoutent à ces définitions des degrés du savoir nager.

Une analyse des différents paliers proposés nous permet de constater une diminution de la distance à parcourir entre les paliers deux 
et trois. Le deuxième niveau est atteint par les enfants ayant l'occasion de recevoir un enseignement de natation pendant leur scolarité du premier degré. Or un quart des enfants scolarisés en France ne bénéficient pas d'un apprentissage leur permettant d'accéder à ce palier. En conséquence, le troisième palier est conçu comme un niveau plancher, un test permettant de déterminer des actions urgentes de soutien. Cetestn'est pas construit dans une logique de continuité d'apprentissage. Il illustre aussi une conception didactique de la nage orientée sur une autonomie adaptative au milieu plutôt que sur une autonomie de déplacement, il en réduit donc l'aspect sécuritaire comme le précise salégende.Au collège, cetest du premier degré du savoir nager a pour vocation de distinguer les nageurs des non-nageurs afin d'établir des actions de soutien; il reste également l'enjeu des acquisitions en fin de collège.

Un dernier palier de cet apprentissage existe, il est nommé " deuxième degré du savoir nager " et est basé sur un déplacement durant un temps de dix minutes associé à six tâches caractérisant une adaptation variée au milieu et une autonomie motrice. Il rejoint les objectifs des programmes collège 2008 en natation longue niveau deux (nager 12 minutes). Cette dernière étape du savoir nager illustre un traitement didactique de l'activité axé sur la santé et la sécurité. Elle exprime l'évolution identitaire de l'EPS, notamment au travers de la mise en place des activités de développement et d'entretien de soi. L'absence de technique de nage supprime son caractère techniciste et sportif. Une approche globale et sécuritaire caractérise ce test et évoque une permanence de cette notion sécuritaire dans la natation scolaire.

Certaines académies distribuent aux élèves des écoles primaires des fascicules et diplômes attestant de la réussite aux différents paliers. Ils sont signés conjointement par l'enseignant d'EPS et par un Maitre-Nageur-Sauveteur diplômé d'État, gage d'une réelle compétence acquise.

Figure 2. Brevet de natation. Rectorat de Paris-2011

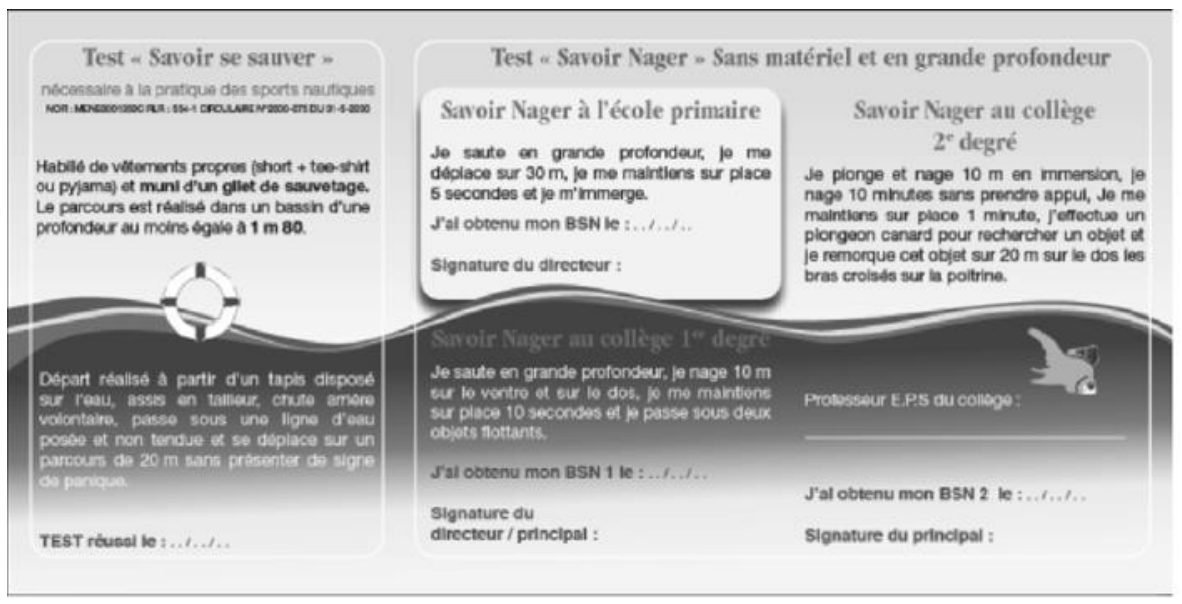

La circulaire de 2011 (circulaire n²011$090 \mathrm{du} 7$ juillet 2011) permet l'ouverture concomitante de bassins à des publics scolaires et non scolaires. D'autre part, la limitation des effectifs est constituée par la surface disponible par élève $\left(\mathrm{m}^{2}\right)$, et la compétence de l'enseignement. L'organisation de l'enseignement de la natation à l'école s'étend ouvertement à d'autres partenaires au travers des structures associatives. Dans le second degré, les élèves en grande difficulté aquatique peuvent bénéficier d'un accompagnement et 
de soutien scolaire, si celui-ci est mis en place localement. L'absence de normes concernant les effectifs réduits des élèves ne sachant pas nager et l'accent porté dans le texte pour des dispositifs particuliers dénotent une transformation radicale de ce paradigme. Si l'autonomie aquatique est un objectif du premier degré de l'enseignement, savoir nager doit être validé au collège, quels que soient les moyens proposés.

La circulaire de 2011 met fin à une stigmatisation de l'enseignement de la natation au regard des autres activités de l'EPS. Ces évolutions semblent contribuer à ce que la natation devienne une activité comme les autres. Elle reste toutefois la seule activité en EPS qui nécessite la présence de surveillants extérieurs à l'enseignement. Force est de constater qu'actuellement nombreux sont les élèves qualifiés comme non-nageurs dans les établissements scolaires (c'est-à-dire n'ayant pas atteint le premier degré du savoir nager). Ceux-ci demeurent une préoccupation du système éducatif dans sa globalité et non plus du fait d'une seule discipline. Les moyens de soutien et d'accompagnement dans un cadre volontaire existent mais le nombre de piscines ne varie pas ou peu et la concurrence de leur utilisation limite de ce fait leur existence. Les actions d'aide et de soutien servent ponctuellement et localement à répondre à ces difficultés d'apprentissage. Ils ne sont que de faibles palliatifs car ils ne possèdent ni le caractère obligatoire ni celui d'un réel suivi.

Tableau 3. Évolution des effectifs de l'enseignement de la natation en EPS, issue des textes officiels de $\mathrm{l}^{\prime} \mathrm{EN}$

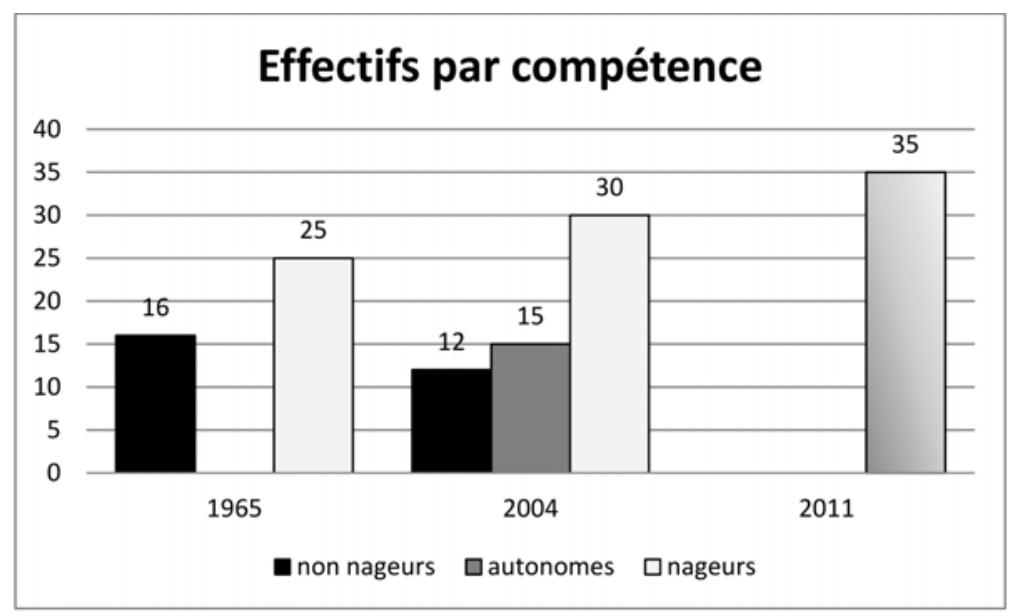

\section{3. discussion}

Si des diplômes fédéraux et des textes officiels de l'Éducation nationale définissent le savoir nager, l'observation des tests proposés depuis 50 ans nous éclaire sur l'évolution des objectifs et des conceptions qui s'y rattachent. On peut différencier deux courants. L'un analytique et techniciste centré sur l'apprentissage d'une technique illustrée par l'apprentissage de la brasse sur une distance définie ( 25 ou 50 mètres) et l'autre global, celui de la familiarisation avec le milieu aquatique illustrant une bonne adaptation, un épanouissement de l'enfant et qui dans une certaine mesure, facilite l'apprentissage de techniques de nage et l'accès aux activités aquatiques.

Concernant la définition du savoir nager, les modèles technicistes se différencient essentiellement dans la distanceà parcourir, 25 mètres 
(soutenu par le texte officiel de 1965), 50 mètres pour être dans le groupe des nageurs à l'école (Circulaire n ${ }^{\circ} 2004-173$ du 15 octobre 2004). Un travail d'harmonisation et de concertation entre maitres-nageurs-sauveteurs et enseignants d'EPS permet d'atteindre des objectifs communs dans la définition d'un savoir nager.

L'apparition de nouvelles pédagogies et de nouvelles théories de l'apprentissage (Piaget, 1972) a trouvé un écho favorable pour répondre à la massification des effectifs scolaires à partir des années 1970. L'éveil aquatique se développe impliquant une prise en compte plus précoce de l'enfant. Il s'agit d'un changement conceptuel, favorisant l'adaptation au milieu et la familiarisation dans le cadre de l'apprentissage. Les modèles éducatifs se sont ainsi éloignés des modèles sportifs. L’intégration de l'EPS au sein de l'Éducation nationale en 1981 atteste de cette approche. La définition du nageur a glissé progressivement vers un modèle global, plaçant, à l'instar de la Loi d'orientation de 1989, l'élève plutôt que le savoir au centre des apprentissages. Une conception globale du savoir nager est déclinée au sein de l'Éducation nationale bien que le test étape n'évalue pas tous les paramètres. La Fédération française de natation fait évoluer dans le même temps ses tests d'apprentissage (ENF) dans une logique également globale. La familiarisation et l'éveil sont pris en charge s'accordant particulièrement avec une évolution des demandes sociétales (Roessle, 2010).

À partir de 2006, la Fédération française de natation (FFN) va modifier sa définition du savoir nager, s'associant aux autres fédérations afin de garder une place centrale dans les activités de la natation mais aussi afin de créer un renouveau. L'Éducation nationale modifie elle aussi progressivement sa définition du savoir nager et ses normes d'encadrement. Diminuant ses prétentions en termes d'autonomie, la définition du savoir nager conserve une conception globale du nageur. L'Éducation nationale ajoute une dimension d'hygiène et de connaissance du milieu qui correspond à une évolution des pratiques physiques des Français orientées vers les activités de pleine nature, les pratiques ludiques et de santé.

Une transformation fondamentale des exigences en termes de distance et de tâches à exécuter démontre lincorporation progressive de toutes les dimensions de l'eau et de l'individu. La FFN passe d'un test de 25 mètres dans une technique de nage à un test sur 50 mètres associant des tâches variées. Conjointement à l'évolution des effectifs scolarisés, l'Éducation nationale s'éloigne d'une conception sportive de l'apprentissage. Elle incorpore une vision globale du nageur, passant d'une définition du nageur sur 50 mètres à une distance de 20 mètres associée à des actions convoquant une adaptation et une connaissance du milieu. La réduction de la distance à franchir nous interroge sur la nouvelle définition de l'autonomie aquatique. Elle confirme l'éloignement des approches sportives, lui préférant l'enchaînement de tâches variées symboles d'une adaptation aquatique. Les termes "savoir nager" sontutilisés dans la scolarité au niveau du troisième palier (Arrêté du 8 juillet 2008). La définition du savoir nager scolaire est associée au concept de surveillance. Ainsi, en 2011, au niveau scolaire est visée l'acquisition d'un " premier degré de savoir nager " au collège illustrant la compétence de savoir nager " sous surveillance". Force est de constater que les différentes instances se préoccupant de l'enseignement de la natation se sont rejointes lentement dans une conception d'un savoir nager, pour des raisons humanistes (accessible au plus grand nombre), culturelles (acquisition d'un patrimoine commun) et sociales. Ainsi, le savoir nager sportif se rapproche du savoir nager scolaire. Toutefois, il parait incongru qu'au regard de ces trajectoires communes des conceptions, un enfant ayant une capacité reconnue à savoir nager au sein de l'Éducation nationale soit considéré comme non-nageur dans le monde fédéral. En effet, l'objectifdans ces deux milieux est le même. Il 
s'agit, au travers d'une culture aquatique commune (Pelayo \& Terret, 1994), de permettre à tous les nageurs d'acquérir ce savoir minimal et fondamental. Bien qu'un test anti-panique (Circulaire n² 2000-075 du 31 mai 2000) existe au sein de l'Éducation nationale, l'acquisition du savoir nager permetl'accès aux différentes pratiques aquatiques et nautiques de loisir; il permet l'entretien de la santé et contribue à développer un aspect sécuritaire de la pratique. Il atteste d'une véritable maitrise aquatique. Une liaison entre les paliers du savoir nager del'Éducation nationale etle sauv'nage est donc nécessaire. Le sauv'nage se situe entre le premier degré du savoir nager (palier trois) et le deuxième degré du savoir nager (palier quatre). Le premier degré du savoir nager nécessite un déplacement sur une distance de 20 mètres, c'est un test essentiel à la détermination d'action de renforcement mais insuffisant comme définition d'un niveau d'autonomie réel en fin de collège. Les tests proposés pardes auteurs comme "le pechomaro " ou définis par les fédérations commele "sauv'nage " sont réalisés autour d'un parcours de 50 mètres nécessitant d'avoir résolu les problèmes liés à la respiration, mais aussiàla propulsion. Ces tests ne constituent pas une réelle nouveauté historique ; Hébert définit en 1959 le savoir nager par une liste de capacité : "flotter sans mouvements ou avec mouvements, réduits, pouvoir franchir une distance, évoluer de diverses façons en surface et en plongée, sauter par la tête ou les pieds, assurer sa propre sécurité, porter secours, exécuter ce qui précède en étant habillé, faire face aux incidents et dangers des eaux en pleine nature " (Terret, 2006, p. 90). L'enchaînement des différentes actions révèle une réelle adaptation au milieu aquatique et leur confère une légitimité sécuritaire que tous les élèves devraient se voir valider. Il est intéressant de noter que l'existence du test " pechomaro " et sa présence dans les textes officiels de 2004 à titre d'exemple ont contribué à la construction par le Conseil interfédéral des activités aquatiques (CIAA) d'un test similaire appelé " sauv'nage " composé également d'un enchaînement de huit tâches sur 50 mètres. Ce testa été expérimenté auprès de nageurs valides et de nageurs en situation de handicap. Bien que d'autres tests soient proposés (Durali \& Fouchard, 2010 ; Bureau et al., 2011), nous pouvons considérer que le test $d u$ "sauv'nage ", à travers sa reconnaissance institutionnelle et sa conception globale de l'apprentissage et de l'autonomie, plaide pour devenir le test de référence du savoir nager en France dans l'optique de pouvoir s'ouvrir sur une polytechnique motrice en matière de loisirs aquatiques et nautiques et devrait pouvoir être validé lors des cours d'EPS.

\section{Conclusion}

Les conceptions de l'enseignement de la natation en France ont évolué historiquement au gré des influences culturelles, sociales et pédagogiques. L'évolution des conceptions (globales, analytiques) de l'enseignement de la natation s'est faite au regard de finalités variées (hygiéniste et santé - sécurité et sauvetage sportive et technique - propédeutique). Le développement des pratiques aquatiques en France peut être considéré comme des marqueurs culturels de cette évolution. Ainsi, on peut dire que la multiplication des formes et usages aquatiques a contribué à inscrire la définition du savoir nager dans une logique d'adaptation motrice au milieu. De la même façon, la recherche identitaire de l'EPS à l'interface d'une culture scolaire et d'une culture sportive représente un élément non négligeable de l'évolution des conceptions et des pratiques des enseignants. De fait, la définition du savoir nager s'est éloignée de ses attributs sportifs au profit d'une éducation motrice et citoyenne. D'une façon similaire, la mise en place au niveau fédéral du CIAA et de ses actions (Sauv'nage, ENF, savoir nager) exprime une volonté d'intégrer les évolutions des 
pratiques et la manne de pratiquants. Ces actions traduisent une évolution des conceptions de la définition du savoir nager axée sur une adaptation aquatique variée.

La natation, longtemps considérée comme un sport de base (Terret, 1999) de l'éducation des enfants au regard du contexte géographique delaFrance, ses côtes et ses rivières, du développement des activités de loisir, mais aussi du rayonnement sur la scène internationale de la natation sportive française, demeure une priorité du ministère des Sports et de l'Éducation nationale. Les moyens et les projets développés attestent de la prise en compte des enjeux du savoir nager. Les différents éléments qui structurent l'enseignement comme la définition du savoir nager premier degré au sens d'une autonomie sous surveillance et l'objectif minimal d'un socle commun prêtent à confusion en termes d'objectif. L'éducation vers une réelle indépendance aquatique semble un enjeu et un objectif à replacer au cœur du débat.

La formation des personnels est également touchée par ces changements. Si les personnels chargés d'assurer l'enseignement de l'éducation physique et sportive doivent justifier, avant leur recrutement, de leur qualification en sauvetage aquatique et en secourisme. (Décret $n^{\circ}$ 2004-592 du 17 juin 2004), la pratique de la natation quant à elle n'est plus obligatoire au concours d'aptitude au professorat d'éducation physique. De la même façon, la pratique de la natation au concours de recrutement d'enseignants du premier degré n'est plus obligatoire, elle devient exigible sous forme d'une attestation de 50 mètres. On peut percevoir par ces dispositions que les objets de formation ne sont pas de nature aquatique.

L'enjeu de cet article était de montrer que si la pratique de la natation est développée en France, son organisation et son enseignement illustrent l'intérêt qu'on lui porte. L'histoire des tests relatifs au savoir nager traduit bien une évolution des pratiques mais aussi une volonté politique dont les prérogatives se reflètent de tout temps, tant dans les objectifs assignés et les moyens alloués, que dans la formation de ses enseignants.

\section{textes de l'éducAtion nAtionAle}

Arrêté du 18juin 1996:B.0. n²9 du 18juin 1996 Arrêté du 14 novembre 1985, paru au BO du 12 décembre 1985

Arrêté du 8 juillet 2008, J.O. du 5 août 2008, paru dans le bulletin officiel spécialn ${ }^{\circ} 6 \mathrm{du}$ 28 août 2008. Les programmes EPS collège. Bulletin Officiel(B.O.) n 10 du 20 juin 1959

Bulletin Officiel (B.O.) n 6 du 11 février 1965 Bulletin Officiel(B.O.)n ${ }^{\circ} 28$ du 14 Juillet2011 circulaire 253 du 18 juillet 1965, paru au B.0. $\mathrm{n}^{\circ} 65-154$ bis du 18 octobre 1965

Circulaires du 23 décembre 1971 et du 9 juin 1972 (71441 et 71286B)

Circulaire du 9 septembre 1971 (Comiti)

Circulairen ${ }^{\circ} 2000-075$ du 31 mai2000 : testantipanique de natation

Circulaire $n^{\circ} 2004-13$ du 13 juillet 2004, parue au B.O. n 32 du 9 septembre 2004, modifiée par la circulaire $\mathrm{n}^{\circ} 2004-173 \mathrm{du} 15$ octobre 2004, parue au B.O. n 39 du 28 octobre 2004

Circulaire n² 2006-197 du 30 novembre 2006

Circulaire n 2010-191 du 19 octobre 2010, parue au B.0 .du 11 novembre 2010

Circulaire n ${ }^{\circ}$ 2011-090 du 7 juillet 2011

Décret n 2004-592 du 17 juin 2004

Décret du 11 juillet 2006

ENSEPS (1973). L'homme et l'eau de l'apprentissage des nages à la maîtrise du milieu aquatique. Châtenay-Malabry.

Loin ${ }^{\circ} 75-620$ du 11 juillet 1975:LoiHaby Loi n 89-486 du 10 juillet 1989 : Loi Jospin Loi n 95-836 du 13 juillet 1995 : Le nouveau contrat pour l'école.

Loi n 2005 -380 du 23 avril 2005 : Loi Fillon

Programmation des APS dans les établissements scolaires du second degré, 3 mai 1967, ministère de l'Éducation nationale et ministère de la Jeunesse et des Sports. 


\section{Bibliographie}

Andrieu, g. (1990). L'EP au XX' ${ }^{\mathrm{e}}$ siècle. Les Cahiers de l'EPS. Joinville.

ARnAud, P. (1989). Les rapports du sport et de l'éducation physique en France depuis lafin duXIX ${ }^{\mathrm{e}}$ siècle, in Éducation physique et sport en France 1920-1980. Paris:Éditions AFRAPS, p. 251-267.

ArtiagA, I. (2007). Culture(s) sportives(s), loisirs et temporalités. Des trois semaines de congés payés aux jours de RTT(1956-1998), in P.Tetart, Histoire du sporten France, delalibération à nos jours. Paris : Musée national du sport-Vuibert, p. 107-117.

AuvRay, e. (2010). Une histoire des pratiques pédagogiques liées à l'enseignement de la natation scolaire entre 1945 et 1995 ( $2^{\text {nd }}$ degré). Carrefour de l'éducation, 2, 30, p. 149-167.

BuReAu, P.- P., buRy, V., mAdelenAt, g., tAveRnieR, J.-C., \& vanRoose, P. (2011). Activités aquatiques. Water-polo, natation synchronisée, natation subaquatique, sauvetage. Paris : Éditions Revue EP.S.

CAllède, J.-P. (2000). Les politiques sportives en France : éléments de sociologie historique. Paris : Economica.

CAtteAu, R. \& géRARd, g. (1968). L'enseignementdelanatation. Paris: Vigot Frères.

CAtteAu, R. (1999). L'enseignement de la natation, in Actes du colloque des 13 et 14 novembre 1997, Paris : Les Cahiers de l'INSEP, 28, p. 207.

clement, J.-P. (1994). Les mutations de la société française et l'évolution du Sport de 1945 à nos jours In P. Arnaud, Éducation physique et sport 1920-1980. Clermont-Ferrand: Éditions AFRAPS.

de maximi, A. (2004). Les réflexes anti-noyade. Prévention et protection pour lutter efficacement contre les noyades. En ligne : www.inpes.sante.fr/70000/ dp/04/dp040506.pdf.

duRAli, s. \& fouchARd, I. (2010). Le guide du savoir-nager. Paris : Éditions Revue EP.S

duRing, b. (1981). La crise des pédagogies corporelles. Paris: Scarabée-CEMEA.

eRARd, c. \& cAtteAu, A. (2008). Les trois modèles d'analyse de l'enseignement de la natation de Raymond Catteau (1950-2006) : influences pédagogiques et scientifiques, in L. Munoz, Usages corporels et pratiques sportives aquatiques du XVIII e au XXe siècle. Tome II. Paris:L'Harmattan, p. 101-123.

fReidlAnd, J., SAvy, m. \& tAlAndieR, m. (2009). Étatdeslieux des bassins de natation en France. Paris : Ministère de la Jeunesse et des Sports.

gAl-PetitfAux, n. (1993). Savoirnager: une pédagogie dela natation. Paris : Éditions Revue EP.S.
gARnieR, P. (1995). Ce dont les enfants sont capables: marcherXVIII ${ }^{e}$, travailler XIX ${ }^{e}$, nager XX ${ }^{e}$, Paris : Métailié.

gARnieR, P. (2002). Enseigner l'éducation physique à l'école élémentaire, Revue STAPS, 57, p. 7-20.

goiRAnd, P., JouRnet, J., maRsenAch, J., moustARd, R., \& PoRtes, m. (2004). Les stages Maurice Baquet 1965 1975 : genèse du sport de l'enfant. Paris : L'Harmattan.

gouRson-VeRgeR, n. \& VeRgeR, m. (1994). Natation : parcours aquatique d'évaluation. Revue EP.S, 249.

gResseR, b. (2005). Clubsdeplaged'hieràaujourd'hui.Thèse de doctorat. Bordeaux. Université de Bordeaux II.

mAillard, d. \& PelAyo, P. (1994). Natation et APPN :le test pechomaro: un savoir nager sécuritaire. Revue Éducation Physique \& Sport, 250, p. 17-20.

mAuss, m. \& lévi-StRAuss, c. (1950). Sociologie et an thropologie. Paris: Presses universitaires de France.

meRle, P. (2002). La démocratisation de l'enseignement. Paris, La Découverte.

meRle, P. (2008). Politiques et aménagements sportifs en région stéphanoise. Paris : L'Harmattan.

mesteJAnot, d. (1992). Le savoir-nager. Analyse comparative enseignants/élèves. Reoue Éducation Physique \& Sport, 237, p. 53-56.

michel, J. (1942). Savoir nager, manuel pratique, conforme aux épreuves des brevets scouts de nageur et sauveteur. Cahors: Éclaireurs Unionistes.

muller, I. (2005) La pratique des activités physiques et sportives en France. Paris : Ministère de la Jeunesse, des Sports et de la Vie associative \& INSEP.

PelAyo, P. \& teRRet, t. (1994). Savoirs et enjeux relatifs à la natation dans les instructions et programmes officiels, Revue STAPS, 33, p. 82-95.

Pelayo, P., maillard d., \& RozieR, d. (2000). De la natation au collègeetaulycée. Paris: Éditions Revue EP.S.

PiAget, J. (1972). Où va l'éducation ? Paris : Gonthiers Denoël, coll. "Médiations ".

Roessle, s. (2010). Construction de dispositions didactiques chezlejeuneenfant, thèsededoctorat. Rennes.

Schoebel, é., RAude, e., \& bécARt, e. (1952). La natation en coloniedevacances. Paris: Bourrelier.

SobRy, c. (2004). Le tourisme sportif. Villeneuve d'Ascq : Presses universitaires du Septentrion.

Soulé, b. \& coRneloup, J. (2007). La conceptualisation en sociologie : influences paradigmatiques et implications méthodologiques, Bulletin de méthodologie sociologique, 93.

teRRet, t. (1998). L'institution et le nageur : histoire de la Fédération française de natation, 1919-1939. Lyon : Presses universitaires de Lyon. 
terRet, t. (1999). Éducation physique et sport de base. InJ. Gleyse et al., L'éducation physique au XXe siècle:approchehistoriqueetculturelle(p. 127-138). Paris:Vigot.

teRRet, t. (2002). L'Éducation physique en France sous la quatrième république. Review Human Kinestics publishers, 35, p. 51-72.

terRet, t. (2006). Natation et méthode naturelle. Movement ESportSciences, 3, n5 59, p. 83-98.

touchARd, y. (2004). Activités aquatiquesetnatation àl'école. Paris : Édition Revue EP.S, 1,119.
vadePied, A. (1976). Laisser l'eau faire. Paris. Éditions du Scarabée.

vAllet, J. (1999). L'enseignement de la natation, in Actes du colloquedes 13et14 novembre 1997. Paris: Les Cahiers de l'INSEP, 205.

Viger, e. (2007). Leseffets dela démocratisation del'enseignementen France: uneétude empirique. Thèse SES, Paris. 